\title{
Erratum: Chimera patterns in two-dimensional networks of coupled neurons [Phys. Rev. E 95, 032224 (2017)]
}

Alexander Schmidt, Theodoros Kasimatis, Johanne Hizanidis, Astero Provata, and Philipp Hövel

(Q) (Received 11 December 2019; published 23 December 2019)

DOI: 10.1103/PhysRevE.100.069901

Equations (2a) and (2b) of this paper were printed incorrectly: The order of variables in the differences of the coupling term were wrongly stated. However, none of the results or conclusions change due to this misprint, since the correct formulas were used in the simulations. The equations should read

$$
\begin{aligned}
\epsilon \frac{d x_{i j}}{d t} & =x_{i j}-\frac{x_{i j}^{3}}{3}-y_{i j}+\frac{\sigma}{N_{r}-1} \sum_{(m, n) \in B_{r}(i, j)}\left[b_{x x}\left(x_{m n}-x_{i j}\right)+b_{x y}\left(y_{m n}-y_{i j}\right)\right], \\
\frac{d y_{i j}}{d t} & =x_{i j}+a+\frac{\sigma}{N_{r}-1} \sum_{(m, n) \in B_{r}^{\mathrm{FHN}}(i, j)}\left[b_{y x}\left(x_{m n}-x_{i j}\right)+b_{y y}\left(y_{m n}-y_{i j}\right)\right],
\end{aligned}
$$

The authors would like to thank A. Ferikoglou for bringing this misprint to our attention. 